Juliette Prudhomme

\title{
Les personnages bibliques, héros d'une épopée grecque chrétienne dans la poésie de Grégoire de Nazianze
}

Dans le corpus poétique de Grégoire de Nazianze, l'influence de la poésie épique au niveau formel est bien visible : Grégoire écrit de nombreux poèmes en hexamètres dactyliques et il emploie les formes morphologiques et lexicales archaïques issues $\mathrm{du}$ corpus homérique. Les poèmes écrits en distiques élégiaques sont eux aussi fortement influencés par la langue homérique ou post-homérique. ${ }^{1}$ Cet usage correspond bien à l'époque à laquelle Grégoire écrit puisque c'est une forme poétique en vogue au IV ${ }^{\mathrm{e}}$ siècle. Citons pour exemple l'oeuvre des deux Apollinaire de Laodicée, le père et le fils, qui se sont essayés à plusieurs formes poétiques dont l'épopée. Grégoire connaît ces œuvres, qu'il juge hérétiques, et affirme dans une lettre qu'il souhaite que ses propres œuvres poétiques se substituent à celle des Apollinaire. $^{2}$

Pourtant, comme la majorité des chrétiens, Grégoire rejette les sujets de la poésie épique traditionnelle puisqu'il dit dans un des deux poèmes qui constituent son art poétique :

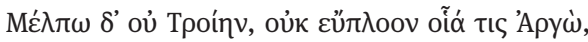

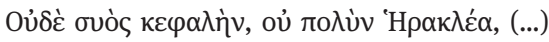

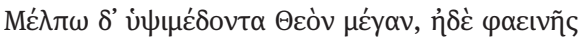

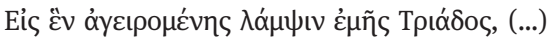

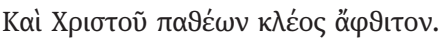

«Je ne chante pas Troie, ni comme un autre Argô à la belle navigation, ni la tête de sanglier ni le puissant Héraclès (...). Je chante le grand Dieu au trône sublime ainsi que la splendeur de ma Trinité brillante, réunie dans une unité, (...) et la gloire impérissable des souffrances du Christ». ${ }^{3}$

Tout en rejetant les sujets épiques, Grégoire affirme ici sa volonté d'écrire des vers épiques et chrétiens. Quelle est donc la place des personnages bibliques dans cette épopée chrétienne ? Si les personnages bibliques servent très souvent d'exempla, comme le montrent très bien les travaux de K. Demoen, peu de longs passages ou de poèmes sont entièrement consacrés à un personnage biblique précis. ${ }^{4}$ Ces pièces existent toutefois : il s'agit essentiellement des poèmes qualifiés de «bibliques», qui évoquent les textes scripturaires et les résument. Ils concernent très majoritairement

1 Ces poèmes se distinguent formellement assez peu des poèmes en hexamètres dactyliques. Voir Prudhomme (2006) 99-103 (http://www.theses.fr/2006LYO20054).

2 Grégoire de Nazianze, Lettre 101, 71, p. 68-69, Paris, 1974 (SC 208).

3 II.1.34, v. 71-72; 77-78; 83 (PG 37, 1307-1309). Traduction personnelle.

4 Demoen (1996).

https://doi.org/10.1515/9783110687224-019 
Jésus et les miracles qu'il a accomplis : le premier est en distiques élégiaques et les trois suivants sont en hexamètres dactyliques. ${ }^{5}$ Un autre poème présente des parallèles intéressants et porte sur les miracles d'Elie et Elisée : il est écrit en trimètres iambiques et va nous permettre d'élargir notre propos aux personnages de l'Ancien Testament. $^{6}$

Ces poèmes courts, peut-être destinés à être appris par cœur par des catéchumènes, ont-ils un intérêt sur le plan littéraire ? L'emploi de la forme et de la langue homérique est-il uniquement formel ou Grégoire cherche-t-il à donner un souffle épique aux héros bibliques ? Pour répondre à ces questions, nous allons procéder à une analyse précise des poèmes cités et des extraits d'autres poèmes qui nous ont paru significatifs.

\section{Des personnages bibliques évoqués dans une langue épique}

Chez Grégoire, les pièces à contenu biblique sont, en grande majorité, composées en hexamètres dactyliques et distiques élégiaques, le poète employant une métrique et une langue relativement élaborées, étrangères au matériau biblique lui-même.

\section{A Les liens avec le modèle scripturaire}

Grégoire dispose pour les poèmes étudiés de modèles bien déterminés et sa fidélité au texte scripturaire est grande. Ainsi, dans le poème Les Miracles du Christ selon Matthieu, Grégoire conserve l'ordre dans lequel sont rapportés les miracles dans chaque évangile. Ce premier poème, qui reprend l'Évangile de Matthieu, est un peu plus long, en raison de l'abondance des miracles qui y sont rapportés. Les deux poèmes écrits selon les Évangiles de Luc et de Marc sont plus courts : ils ont un nombre de vers à peu près semblable et comportent de très nombreux points communs. ${ }^{7}$ En revanche, le poème qui reprend l'Évangile de Jean est nettement plus court et ne fait que onze vers, ce qui est conforme au matériau biblique d'origine.

5 Quatre pièces sont intitulées Sur les miracles du Christ et portent sur les miracles rapportés dans le Nouveau Testament, composées, pour trois d'entre elles, en hexamètres dactyliques, et pour l'une en

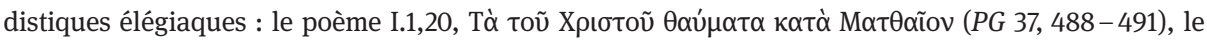

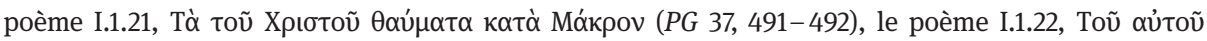

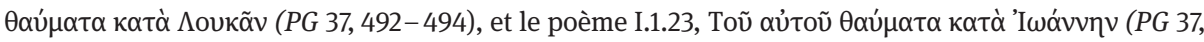
492-494).

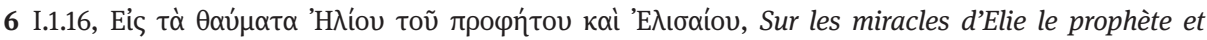
d'Elisée (PG 37, 477-479).

7 Une seule confusion apparaît à la fin du poème 21, où il est question des aveugles et des boiteux, épisode que Grégoire rattache à l'Evangile de Marc alors qu'il apparaît dans celui de Matthieu. 
L'étude du lexique montre aussi une certaine dépendance des vers de Grégoire à l'égard du texte scripturaire. Dans le poème Les Miracles du Christ selon Matthieu, plusieurs termes scripturaires sont conservés, même si Grégoire change parfois légèrement leur forme ou leur fonction. ${ }^{8}$

Dans quelques cas, on constate que Grégoire utilise un terme qui est attesté dans le texte scripturaire, mais qui apparaît dans un autre passage que celui qu'il est en train de paraphraser. Ce phénomène est perceptible dans les Miracles du Christ selon

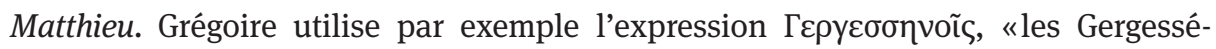
niens» (20, v. 7), terme attesté dans l'Évangile de Luc et non dans celui de Matthieu. Les démons sont souvent désignés par le terme $\Delta \alpha \dot{\prime} \mu \omega v(20$, v. 7, v. $12 ; 21$, v. 3 ; 22, v. 3 et v. 13) qui n’apparaît pas dans les passages paraphrasés mais qui est bien attesté dans le Nouveau Testament (Mt 8.31; Ap 16.14). Il est possible que Grégoire préfère ce terme en raison de sa présence dans le lexique épique. Le terme $\delta \alpha \mu$ ovíov (20, v. 14) est lui aussi emprunté à un verset relatif à un autre épisode que celui paraphrasé (Mt 9.33).

\section{B Le recours à la langue épique profane}

Même si Grégoire s'éloigne peu des Evangiles dans l'exposé des miracles, il recourt à un lexique qui est spécifiquement poétique et qui n'est pas attesté dans les passages bibliques correspondants. Dès une première lecture, le lecteur perçoit une différence entre la langue des poèmes en distiques élégiaques et hexamètres dactyliques d'un côté, et celle du poème en trimètres iambiques de l'autre. Dans les pièces en distiques élégiaques et hexamètres dactyliques, Grégoire utilise des désinences épiques

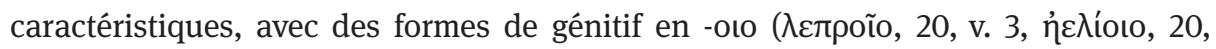

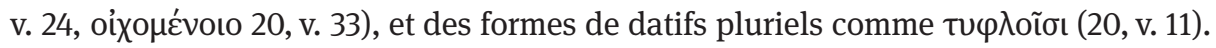
Grégoire utilise plusieurs fois des formes ioniennes comme voṽ $\sigma o v(20, v .3), v \tilde{n} \alpha$ (20, v. 17), vпoũ (20, v. 34). Plusieurs termes sont absents de l'Ancien Testament mais attestés en poésie épique : ainsi, le poète substitue ici au terme $\pi \varepsilon v \theta \varepsilon \rho \alpha ́$ (Mt 8.4) qui désigne la belle-mère dans les Evangiles, le mot homérique غ̇кupá (20, v. 5). ${ }^{9}$

De manière plus intéressante, il apparaît que Grégoire passe du lexique biblique à celui de la poésie profane, sans difficulté apparente. Ainsi, pour évoquer la guérison de la main sèche, le poète utilise deux fois l'adjectif łnpós qui est dans les Évangiles, tandis que dans le troisième poème, il emploie un adjectif homérique

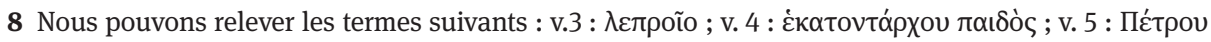

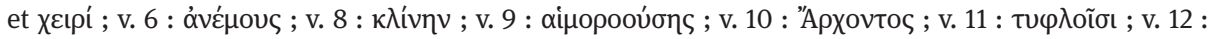

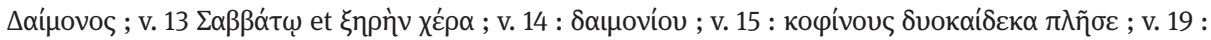

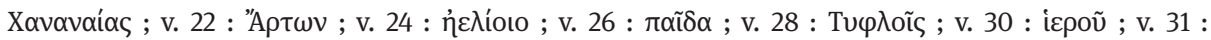

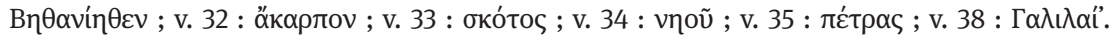

9 Il. XXII.451; XXIV.770.
} 


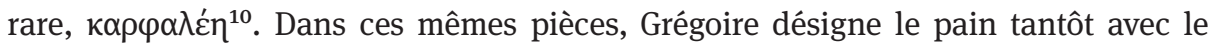
terme des Évangiles, åpтoৎ (21, v. 8 et v. 22), tantôt avec un terme homérique

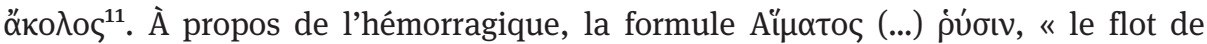

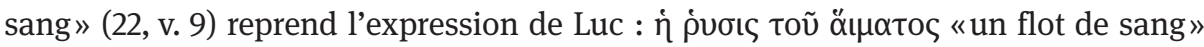
(Lc 8.44), en changeant l'ordre des mots, alors que l'expression $\alpha i \mu \alpha \tau o ́ \varepsilon \sigma \sigma \alpha v ~(. .$.

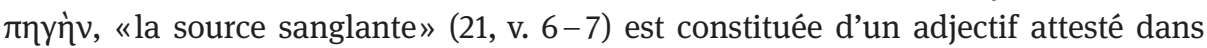
l'Iliade et chez Sophocle ${ }^{12}$.

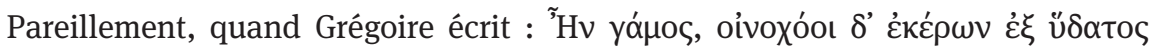
oĩvov, "Il y avait un mariage : les échansons mélangeaient le vin, à partir de l'eau»

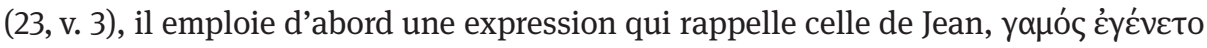
(Jn 2.1), mais change la forme verbale pour former un dactyle. Grégoire substitue ensuite au terme scripturaire $\delta$ ı́́kovoı, «les servants» (Jn 2.5) le terme homérique oivoxóou, «les échansons» et emploie une expression verbale typiquement homéri-

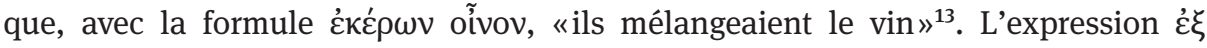

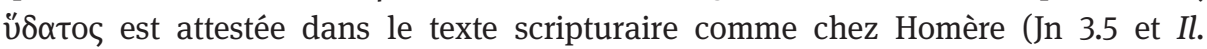
XXII.152) et le terme final, oívov, est placé en fin d'hexamètre, comme chez Homère.

L'examen de ces quelques exemples montre que, si la langue de Grégoire est composée d'éléments hétérogènes, le poète cherche souvent à employer une langue qui a une allure poétique, à varier le vocabulaire, tout en restant proche du texte scripturaire. Il est surtout révélateur de voir que le poète passe avec aisance, dans un même vers, d'une formulation qui nous semble plutôt proche du texte scripturaire à une expression d'allure plus poétique.

\section{Du personnage biblique au héros épique}

Une analyse axée sur les procédés stylistiques fait apparaître que Grégoire ne donne pas seulement un coloris poétique à ses vers mais cherche à mettre en valeur les personnages bibliques en reprenant des caractéristiques propres au genre épique.

\section{A La stylisation de la matière biblique}

Dans les exemples étudiés, il apparaît clairement que Grégoire procède non par amplification, comme de nombreux poètes latins auteurs de paraphrases bibliques, mais par omission. Les poèmes étudiés sont relativement courts, et si le poète reprend quelques éléments clés et caractéristiques, il en omet un plus grand nombre. Ces omissions sont rendues impératives par la brièveté du poème, brièveté qui vise

10 Od. V.369 et Il. XIII.409.

11 I.1.20.16 ; I.1.21.11; I.1.22.10. Voir Od, VII.222.

12 Il. V.82 ; Soph. Ant. 529 ; Anth. Pal. VI.154.

13 Od. XXIV.364 
sans doute à faciliter le travail de mémorisation. Cette brièveté est parfois extrême et conduit Grégoire à adopter très souvent un style concis, voire elliptique. Ainsi, quand

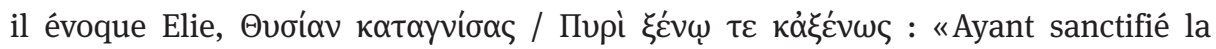
victime par un feu étrange(r) et de manière étrange» (16, v. 7-8), il faut sans doute

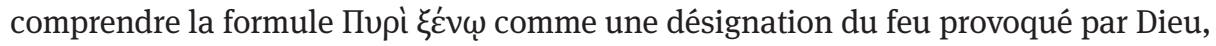
tandis que l'adverbe $\kappa a ̉ \xi \xi \varepsilon v \omega \varsigma$ renvoie à l'ordre donné par le prophète de jeter de l'eau sur le feu (3 reg 18, $30-40$ ).

Dans le poème Les Miracles du Christ selon Matthieu, les omissions sont pareillement très visibles : Grégoire récapitule en effet, dans cette pièce, des éléments épars de l'Evangile de Matthieu et les rassemble sous une forme versifiée et plus condensée. Tous les passages de dialogue disparaissent et seule l'action miraculeuse est rapportée. Souvent, les éléments relatifs au cadre spatio-temporel sont omis, sauf quand ils sont particulièrement significatifs pour l'identification du miracle (v. 7, 13, $28,31,38)$. Ce recours à la concision permet au poète de focaliser l'attention sur la figure évoquée : Jésus ou les prophètes.

\section{$B$ Le héros en action}

Un des éléments stylistiques récurrent qui permet de mettre le héros en valeur est le choix de formes verbales actives, qui n'apparaissent pas dans les passages bibliques paraphrasés. Ce phénomène est bien visible dans les épisodes de guérison, puisque Grégoire recourt à des formes verbales actives ou moyennes, alors que, dans les textes scripturaires, ce sont des formes passives qui sont employées. Grégoire écrit

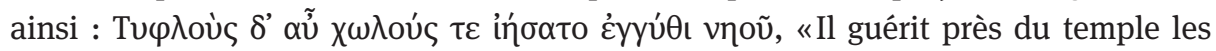
aveugles et les boiteux» (21, v. 17), alors que dans l'Evangile de Matthieu, le verbe est

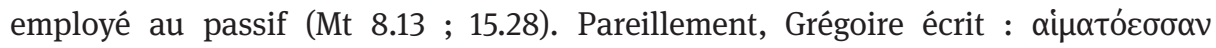

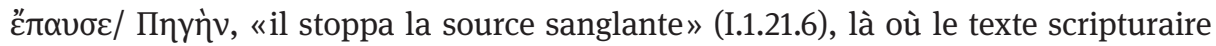

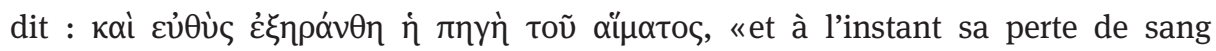
s’arrêta» (Mc 5.29). L'épisode de la fille de Jaïre est évoqué dans deux des poèmes.

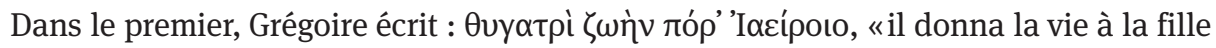

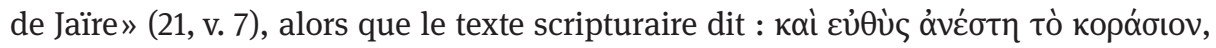
«et aussitôt la fillette se leva» (Mc 5.42). Dans le deuxième poème, la formule :

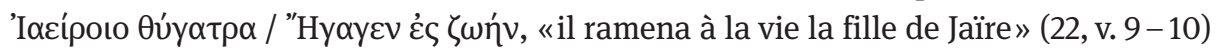

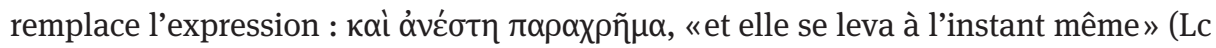
8.55). Si certains verbes sont conservés, Grégoire préfère aux tournures passives des tournures actives, mettant ainsi en valeur la figure du Christ agissant, alors que dans le texte biblique, c'est souvent la personne guérie qui est en position de sujet. Ce procédé permet à Grégoire de changer le point de vue de la narration pour souligner le rôle salvateur du Christ.

Le même procédé apparaît pour les prophètes Elie et Elisée. Ainsi, chez Grégoire, Elie est le maître de la pluie (16, v. 6-7), alors que dans l'Ancien Testament, Elie observe et annonce le retour de la pluie, mais ne la provoque pas réellement. $\mathrm{Pa}$ - 


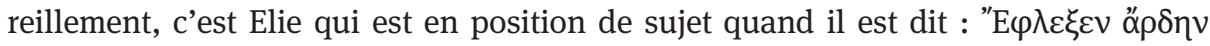

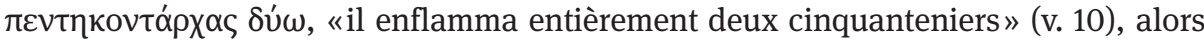

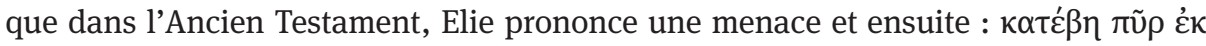

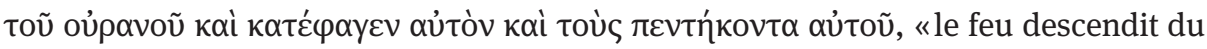
ciel et le dévora, ainsi que ses cinquante hommes» (4 Rg 1.12). C’est Elisée qui de-

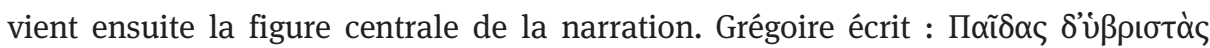

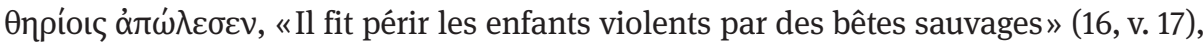

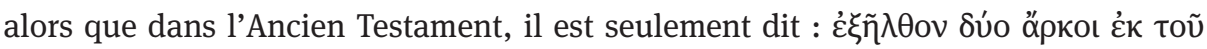

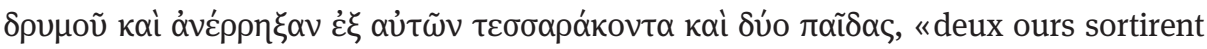
de la forêt et dépecèrent quarante-deux de ces enfants» (4 Rg 2.24). Grégoire focalise donc ici aussi l'attention non plus sur les victimes mais sur la figure prophétique concernée, présentée comme agissante et placée au premier plan.

\section{Le recours aux images poétiques frappantes}

Une autre caractéristique de la réécriture des miracles est le choix par Grégoire d'un lexique plus expressif, qui permet lui aussi de mettre en valeur la figure de Jésus.

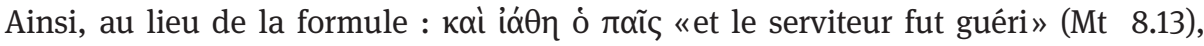

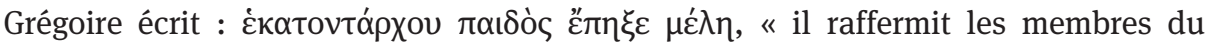
serviteur du centurion» (20, v. 4). La formule $\mu \varepsilon^{\prime} \lambda \eta \eta \dot{\eta} \gamma v v \mu$, «raffermir les membres»,

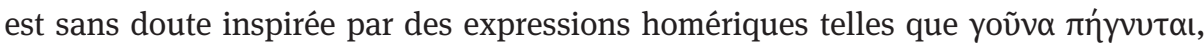
«les genoux se raidissent ${ }^{14}$. Toutefois, alors que, dans ces expressions, le verbe $\pi n ́ y v u \mu$ est employé au passif et évoque la peur qui raidit certains membres, Grégoire l'emploie ici à l'actif et dans un sens positif, afin de bien souligner que l'initiative salvatrice revient au Christ. Dans ce cas, Grégoire joue donc sur un écart de sens avec le modèle littéraire profane, pour souligner la puissance miraculeuse du Christ.

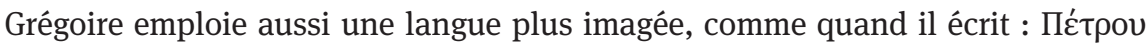

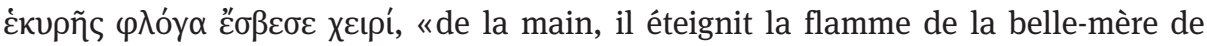
Pierre» (20, v. 5). Le poète réemploie ici une expression prosaïque, déjà appliquée à un phénomène miraculeux, dans un sens métaphorique. ${ }^{15} \mathrm{Il}$ met en outre en valeur le caractère apaisant du geste du Christ, alors que, dans l'Evangile, il est simplement

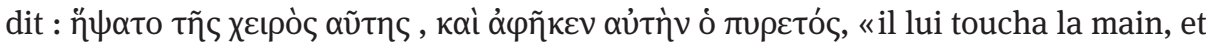
la fièvre la quitta» (Mt 8.15). C'est encore une formule plus imagée qui est employée,

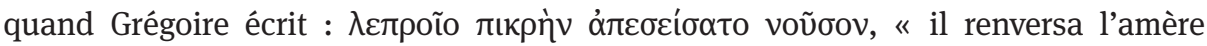

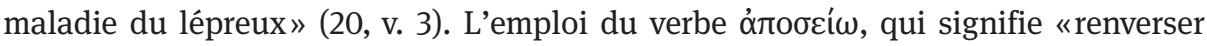
par une secousse», donne au vers l'allure d'un combat en personnifiant la maladie 
comme un ennemi qu'il faut abattre. ${ }^{16}$ Si des emplois métaphoriques sont attestés chez Aristophane avec ce verbe, son usage avec le thème de la maladie semble une innovation de Grégoire.

L'emploi récurrent de la métaphore du lien permet pareillement de mettre en valeur la figure du Christ. Cette métaphore est attestée dans certains passages évangéliques. ${ }^{17}$ Grégoire en a toutefois un usage particulièrement abondant et fait

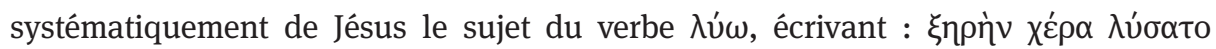
$\delta \varepsilon \sigma \mu \tilde{\omega} \nu$, «il délia une main sèche de ses liens » (20, v. 13), ou encore $\delta \varepsilon \sigma \mu o ̀ v ~ \varepsilon ้ \lambda v \sigma \varepsilon$ /

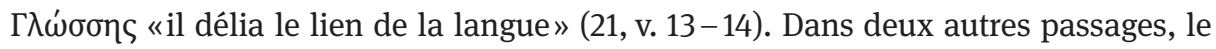

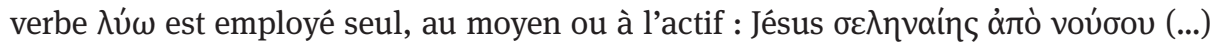

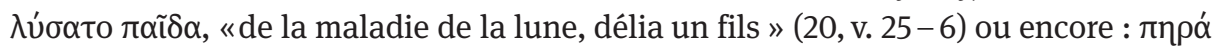

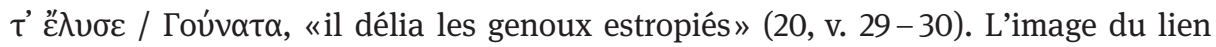
apparaît aussi pour souligner la force du Christ capable de dompter les éléments,

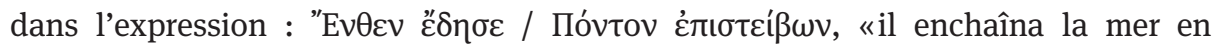
marchant dessus» $(21$, v. 8$)$, scène dans laquelle Jésus est présenté dans sa supériorité par rapport aux forces du mal, incarnées par la mer.

La personnification des éléments opposés permet aussi de faire du Christ un

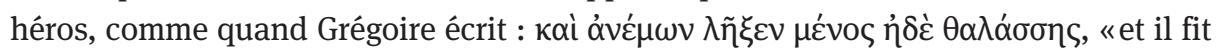

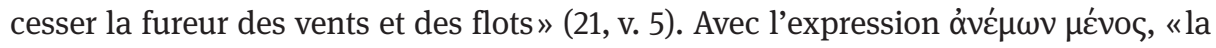
fureur des vents", Grégoire personnifie les forces cosmiques et il crée un tableau

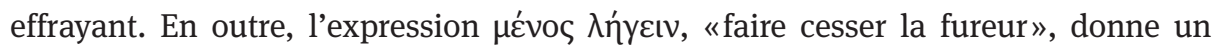
coloris épique à l'expression puisque c'est un emprunt à Homère, pour parler de la colère d'Idoménée qui veut massacrer les Troyens. ${ }^{18}$

Grégoire recourt donc à plusieurs procédés littéraires caractéristiques de l'épopée pour parler de Jésus et des prophètes Elie et Elisée : il met ainsi en valeur ces figures bibliques qui deviennent des héros puissants et salvateurs, évoqués dans de courtes vignettes stylisées.

\section{Une épopée chrétienne}

Dans son projet poétique cité en introduction, Grégoire dit qu’il veut chanter «le grand Dieu» (II.1.34.77) et non plus les sujets épiques habituels, ou encore «la gloire impérissable des souffrances du Christ» (II.1.34.83), formule dans laquelle il emploie

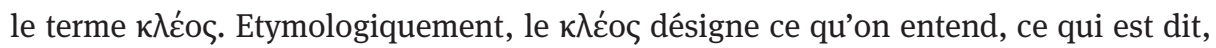
et par la suite signifie la gloire, gloire que la poésie homérique a pour but de chanter. ${ }^{19}$ Grégoire reprend donc la fonction traditionnelle de l'aède mais remplace les héros païens traditionnels par Jésus ou les figures bibliques. Nous avons vu

16 On trouve un emploi poétique de ce verbe chez Theog. Eleg. I.348.

17 Lc 13.16 et Mc 7.35

$18 \mathrm{Il}$. XIII.424.

19 Nagy (1991) 16-17. 
jusqu'à présent qu'une place importante était accordée à la figure de Jésus : nous allons maintenant analyser comment se fait ce travail de substitution en cherchant des parallèles plus précis entre les figures bibliques et les héros épiques traditionnels.

\section{A Jésus comme substitut des héros épiques traditionnels}

Si Grégoire favorise des formulations qui soulignent la puissance du Christ, il n'hésite pas non plus à reprendre des formules qui renvoient non seulement à un contexte militaire mais qui, en plus, désignent l'action d'un héros ou même d'un dieu de l'épopée.

Dans les poèmes portant sur les miracles, pour évoquer le Christ qui «chasse

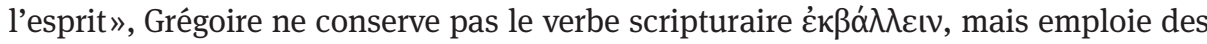

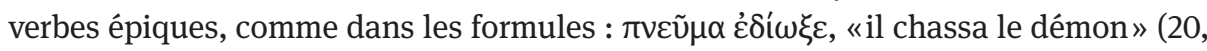

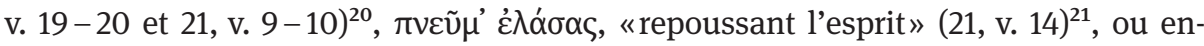

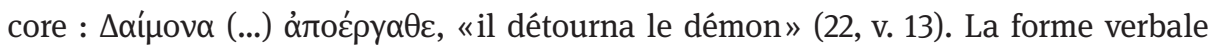

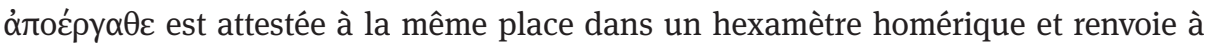
un contexte guerrier dans lequel intervient un dieu : il est en effet question d'un combat entre Achille et Agénor, au cours duquel Apollon, choisissant d'intervenir :

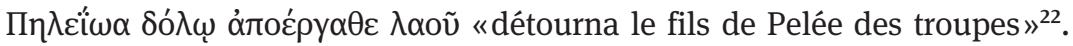

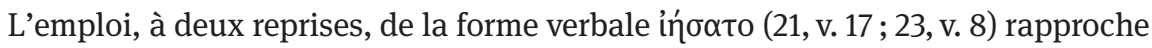
quant à elle le Christ de Péon, le médecin des dieux dans l'Iliade, à propos duquel la

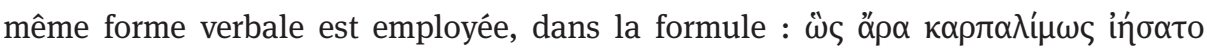

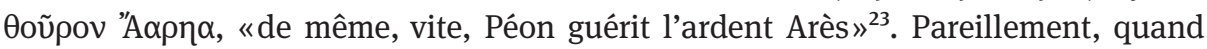
Grégoire dit que Jésus : $\varepsilon \xi \xi \sigma \alpha ́ \alpha \omega \sigma \varepsilon \mu \alpha \theta \eta \tau \alpha$ c,, «sauva ses disciples» (23, v. 7), il reprend une forme verbale homérique, employée à propos de Poséidon qui, poursuivant Ajax : $\varepsilon \xi \xi \sigma \alpha ́ \alpha \omega \sigma \varepsilon \theta \alpha \lambda \alpha ́ \sigma \sigma \eta \varsigma$ « le sauva des flots» ou à propos d'Aphrodite qui, pro-

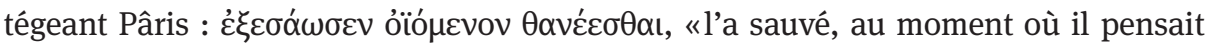
périr ${ }^{24}$. On voit donc que Grégoire évoque Jésus dans les mêmes termes que l'aède évoquait les dieux païens de l'épopée.

Un deuxième parallèle nous semble intéressant. Dans les pièces étudiées, Grégoire choisit un sujet très spécifique, le miracle, qui n'est pas seulement biblique, mais appartient aussi à la littérature grecque profane, et même, à certaines formes

20 Le verbe appartient au vocabulaire guerrier épique, mais n’est pas employé à l'aoriste chez les autres poètes épiques.

21 Voir Il. XVI.87 et XXI.217.

22 Il. XXI.599.

23 Il. V.904.

24 Od. IV.501 ; Il. IV.12. 
poétiques. ${ }^{25}$ Certains hymnes homériques laissent ainsi une grande part aux récits à caractère miraculeux, en particulier l'Hymne homérique aux Dioscures, dans lequel les divinités domptent les vents et la tempête, motif repris dans une idylle de Théocrite. ${ }^{26}$ Les pièces les plus proches auxquelles nous pouvons comparer celles de Grégoire appartiennent à l'Anthologie de Planude et énumèrent les travaux prodigieux d'Héraclès. Le mot «miracle» n'y est pas employé, mais les structures de ces pièces sont semblables à celles des poèmes de Grégoire. Nous citons les premiers vers du poème 92 qui est en hexamètres dactyliques et comporte des adverbes numériques :

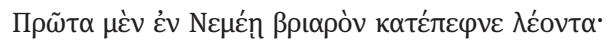

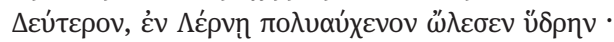

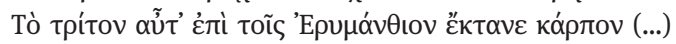

«Premièrement, il massacra le puissant lion à Némée.

Deuxièmement, à Lerne il exécuta l'hydre aux cous sans nombre.

Troisièmement, il tua le sanglier qui hantait l'Erymanthe (...) $»^{27}$.

Si l'on compare cette énumération avec celle du poème 20 , on peut rapprocher le

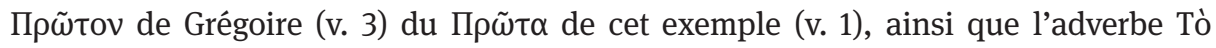
$\tau$ ¡́ítov placé dans les deux poèmes en début d'hexamètre (20, v. 5). Il est possible que Grégoire ait eu connaissance de ce genre de poèmes, consacrés à Héraclès ou à une autre divinité ayant accompli des hauts faits. Cette proximité entre les épigrammes consacrées aux travaux d'Héraclès et les poèmes de Grégoire apparaît aussi au niveau lexical ou thématique. Dans les deux types de poèmes apparaissent, au nombre

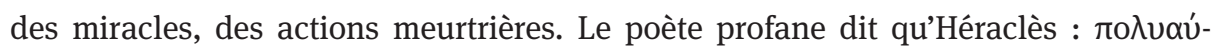

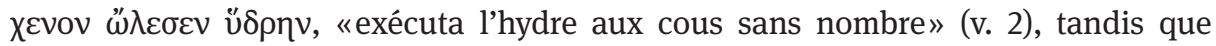

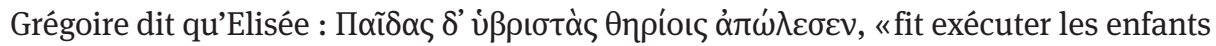
violents par des bêtes sauvages» (16, v. 17). D’autres ressemblances lexicales montrent qu'un terme qui désigne, dans les épigrammes profanes, un travail herculéen, remarquable par la force déployée, est repris par Grégoire pour parler d'une action

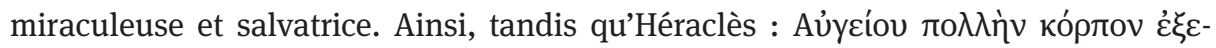

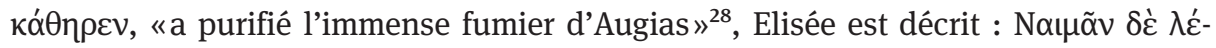

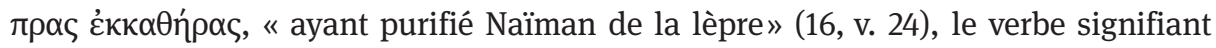
que Naïman est sauvé. Pareillement, le verbe «chasser», qui est employé pour parler

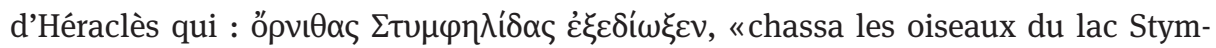

25 Voir à cet égard Cotter (1999), qui est constitué d'un répertoire de textes de l'Antiquité profane et de textes scripturaires. Pour la plupart des miracles présents dans les poèmes cités, elle propose des parallèles. Elle consacre plusieurs chapitres aux miracles concernant la guérison, l'expulsion des démons, le contrôle du vent et de la mer, et le changement de l'eau en vin.

26 Hymne homérique aux Dioscures, v. 12-17 et Theocr. Id. 22, v. 8-22.

27 Anthologie de Planude 92, v. 1-3, Paris, C.U.F., 1980 (trad. R. Aubreton, F. Buffière, p. 114-115).

28 Anthologie de Planude, 92, v. 7. 


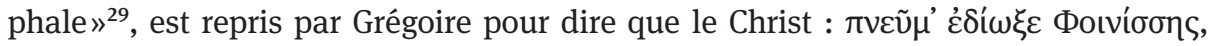
«chassa l'esprit de la femme de Phénicie» (21, v.9), action qui permet là encore une libération et une guérison humaine.

\section{B La christianisation des personnages vétéro-testamentaires}

Un autre procédé d'écriture témoigne de la volonté de Grégoire de créer un héroïsme chrétien : il s'agit de la christianisation des miracles accomplis par les prophètes Elie et Elisée. Pour les prophètes comme pour Jésus, Grégoire emploie le terme $\theta \alpha u ́ \mu \alpha \tau \alpha$ et procède ensuite par énumération. Les formulations choisies pour les prophètes renforcent encore le parallèle entre les miracles.

Ainsi, la reformulation de l'épisode dans lequel Elie redonne vie au fils de la veuve (16, v. 5-6) est particulièrement significative. Grégoire écrit dans son poème :

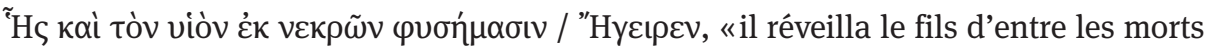
par son souffle» (16, v. 5-6). Avec le terme $\varphi v \sigma n ́ \mu \alpha \sigma ı$, Grégoire conserve l'idée

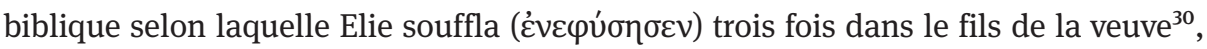

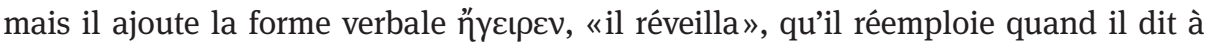

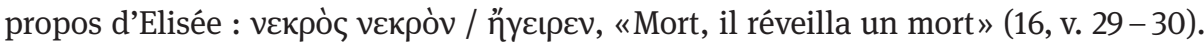

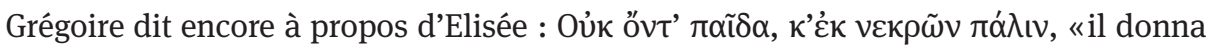
un fils, qui n'existait pas, (le ramenant) ensuite d'entre les morts» (16, v. 21), quand il

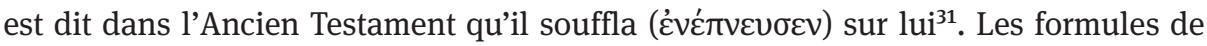
Grégoire rappellent les expressions habituellement employées par les chrétiens pour parler de la résurrection, comme lorsque les évangélistes écrivent à propos de Jean-

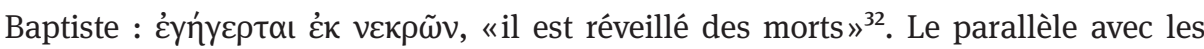
événements néo-testamentaires est renforcé par l'emploi du même type d'expression dans les poèmes rapportant les miracles de Jésus, puisque Grégoire écrit à propos du

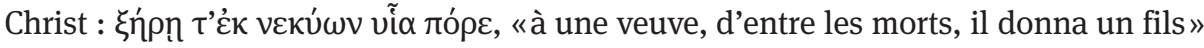
(22, v. 6). Grégoire suggère donc que les prophètes bibliques possèdent une force qui préfigure la résurrection, à la suite d'autres Pères grecs. ${ }^{33}$

Ces quelques exemples montrent bien la volonté de Grégoire de présenter à ses lecteurs des héros chrétiens, substituts aux dieux païens évoqués dans l'épopée, dans un mouvement de surenchère qui en fait des héros exceptionnels.

29 Anthologie de Planude, 92, v. 5.

$301 \operatorname{Rg} 17.21$.

$312 \operatorname{Rg} 4.35$.

32 Mc 6.14, à propos de Jean-Baptiste.

33 Irénée de Lyon évoque ainsi trois épisodes de la vie d'Elie dans leur rapport avec le salut chrétien : le jeûne, la théophanie de l'Horeb, mise en lien avec la Transfiguration, et l'enlèvement sur le char, qui préfigure la résurrection des Justes. Voir Iren. AH III.22.2 ; IV.20.9 -11; V.5.1 ; 21.2. Origène voit dans la traversée du Jourdain par Elie une figure du baptême, (Origène, Sur l'Evangile de Jean VI.238 -51), comme, plus tard, Basile de Césarée (Basile de Césarée, Sur le baptême I.2.5.21). 


\section{Grégoire, un héros de l’épopée chrétienne}

Deux autres extraits, qui s'éloignent des exemples étudiés jusqu'à présent, ont retenu notre attention et permettent de mettre en lumière un procédé caractéristique et original de l'écriture poétique de Grégoire. Les personnages bibliques que Grégoire y évoque - Moïse, Phinéès et Job - lui servent en effet de figures d'identification et lui permettent de se mettre en scène, lui le poète, comme le nouveau héros de son épopée chrétienne.

\section{A Grégoire combattant de la parole, nouveau Moïse et Phinéès}

Dans un poème dans lequel Grégoire exprime ses inquiétudes à voir la Trinité remise en cause, il dresse le portrait de l'homme qui pourrait sauver la situation. Bien que Grégoire ne présente pas ce portrait comme le sien, il est très probable qu'il parle de lui. Il écrit :

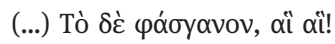

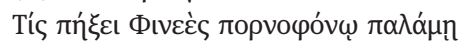

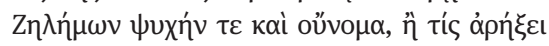

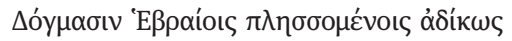

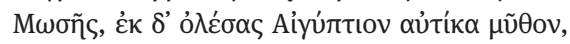

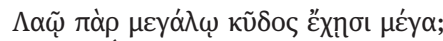

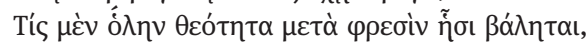

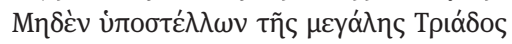

'Hغ̀ vó

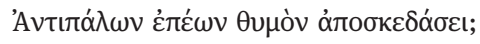

«L'épée ! Hélas! Hélas! Quel est le Phinéès qui la plantera d’une main pourfendeuse de la fornication, plein de zèle pour l'âme et le nom, ou quel est le Moïse qui défendra les enseignements des Hébreux criminellement frappés, et qui, ayant ainsi aussitôt anéanti la fable égyptienne, recueillera une grande gloire auprès de ce grand peuple ? Qui concevra dans son esprit la divinité tout entière, sans rien soustraire à la grande Trinité, ou qui, grâce à un esprit subtil et des paroles vigoureuses, repoussera la colère des déclarations ennemies ? $»^{34}$

Grégoire fait ici référence à deux figures vétéro-testamentaires, qui servent déjà de modèle aux chrétiens avant lui. ${ }^{35}$ Phinéès est un prêtre qui tue un israélite pour le punir de forniquer avec une étrangère, adepte du culte de Baal-Péor. Il les transperce en plein ventre, geste par lequel il fait cesser le fléau qui s'abattait sur le peuple (Nb 25.7-8). Ce personnage est évoqué dans des formules à caractère épique : il est armé

34 II.1.15.21-30.

35 Le statut de modèle de Phinéès apparaît en 1 M 2, 54. Voir également Philon d'Alexandrie, De vita Mosis I, 301 et Grégoire de Nysse pour qui le geste de Moïse est une image du combat de la vraie religion contre le paganisme, Vie de Moïse II.13. 


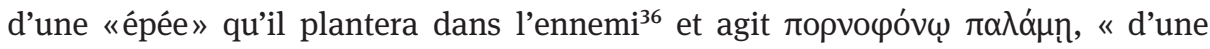
main pourfendeuse de la fornication», expression formée à partir d'un adjectif inventé par Grégoire pour souligner la dimension meurtrière du combat. Avec Moïse, dont le nom est mis en valeur en début de vers, Grégoire se réfère à l'épisode dans lequel celui-ci tue un égyptien qui s'est attaqué à un hébreu (Ex 2, 11-2), acte héroïque que Grégoire interprète selon les codes de l'épopée puisqu'il précise que

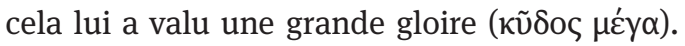

Ces deux modèles, Grégoire les choisit pour évoquer un combat d'un autre ordre : celui qu'il peut livrer grâce à ses paroles. La question posée par le poète au vers

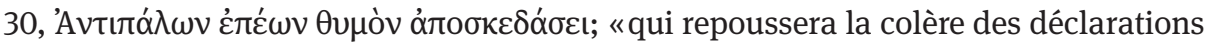
ennemies ?», concerne un combat dogmatique, mais rappelle, formellement, des combats physiques, puisque le verbe est employé habituellement pour parler des soldats. ${ }^{37}$ Les paroles du défenseur de la Trinité sont quasiment personnifiées avec le

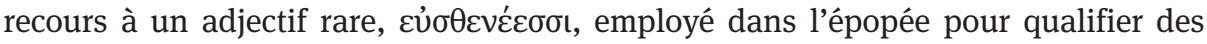

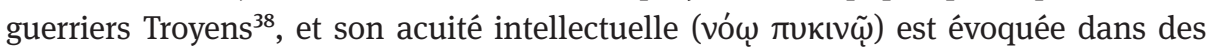
termes employés par Homère pour parler de l'intelligence divine ${ }^{39}$. Pour peindre le portrait du poète justicier, Grégoire utilise donc des figures scripturaires emblématiques du combat contre le mal et l'impiété : il y voit des modèles pour son propre combat et devient ainsi lui-aussi un héros épique au service de la Trinité

\section{B La gloire paradoxale : «je suis un nouveau Job»}

Une autre figure biblique à laquelle Grégoire s’identifie est celle de Job, personnage grâce auquel il réinterprète le motif épique de la gloire, déjà présent dans le passage précédent. Si Grégoire dit vouloir chanter la gloire du Christ, il se met aussi lui-même en scène comme un sujet poétique, digne d'être chanté car accablé de maux. Il introduit donc une nouvelle idée : celle de la gloire paradoxale. Dans ce poème, Grégoire déclare :

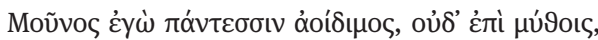

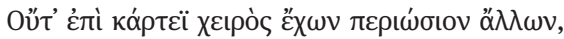

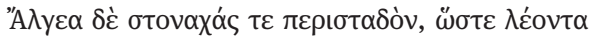

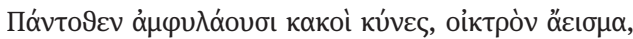

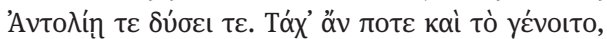

"H

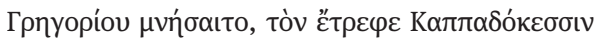

36 Le terme $\varphi$ óoyavov est employé par Sophocle pour parler de Déjanire dont le flanc est transpercé

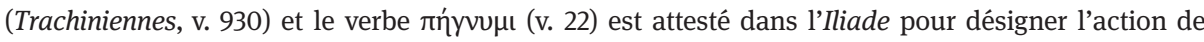
planter une pique : Il. XXII.283

37 Voir par exemple Démosthène, Sur la couronne, v. 289.

38 Quintus de Smyrne, Suite d'Homère I, v. 232. Voir aussi Suite d'Homère IV, v. 44 et v. 293.

39 Il. XV.461. 


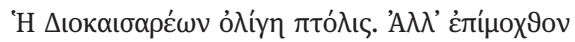

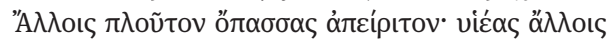

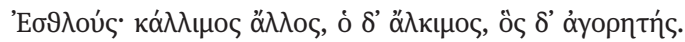

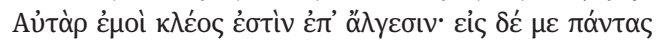

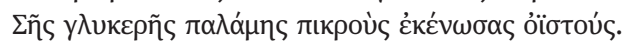

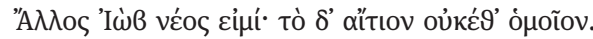

«Moi seul je suis digne d'être chanté par tous, non parce que je dépasse les autres par mes paroles ou par la force de mon bras, mais parce que je suis entouré de souffrances et de gémissements, comme un lion qu'environnent de toutes parts en aboyant des chiens méchants, misérable objet de chansons du Levant au Couchant. Il pourrait bien se faire qu'un homme (...) fasse mémoire de Grégoire qu'a nourri en Cappadoce la petite cité de Diocésarée. Mais à certains, tu as accordé l'infinie richesse laborieuse, à d'autres de nobles fils ; celui-ci est beau, celui-là est vaillant et cet autre bon orateur. Mais moi, ma réputation repose sur mes souffrances. Sur moi, tu as vidé toutes les flèches amères de ta douce main. Je suis un autre Job, mais la cause n'est plus la même $»^{40}$.

À l'image de son modèle, Grégoire se décrit comme un lion environné de méchants (Jb 10.16), ou comme une cible sur laquelle Dieu envoie ses flèches (Jb 16.12). Le paradoxe vient de ce que contrairement à Job, ses souffrances extrêmes ne lui apportent pas la gloire, mais font seulement de lui un motif de chansons. Pour ex-

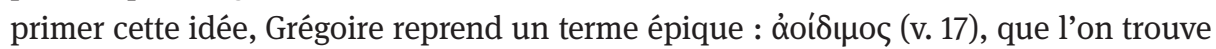
dans l'Iliade ${ }^{41}$, et qui signifie à l'origine, selon J. P. Vernant, «devenir digne d'un chant qui raconte, dans une geste sans cesse reprise et répétée, un destin admiré de tous». ${ }^{42}$

Grégoire insiste sur le caractère paradoxal de sa gloire, et l'oppose aux conceptions épiques en inversant un certain nombre de motifs. Ainsi, il évoque l'étendue géographique de sa gloire qui correspond, en négatif, à celle des héros de l'épopée. ${ }^{43}$ La formule employée par Grégoire, «du Levant au Couchant» (v. 21), placée en début de vers, rappelle des épigrammes écrites en l'honneur d'un grand personnage devenu célèbre à travers le monde. ${ }^{44}$ Grégoire reprend ici une manière de s'exprimer qui souligne habituellement l'ampleur de la gloire des empereurs, pour évoquer sa propre gloire, dont la grandeur repose sur des attributs opposés.

Pareillement, quand Grégoire énumère plusieurs types de personnages glorieux, il établit une liste qui reprend le procédé rhétorique du priamel, présent dans l'Iliade. ${ }^{45}$ Le poète chrétien compare en effet sa situation à celles qui, traditionnellement, sont synonymes de gloire : la richesse, l'abondante descendance, l'éloquence,

40 II.1.19.17-22; 25-31 (PG 37,1271-1273)

41 Il. VI.357-358.

42 Vernant (1989) 93.

43 Od. I.344.

44 Voir l'épigramme consacrée à Constantin dans Anthologie de Planude 369, v. 1-3 et l'épigramme 64, consacrée à Néron.

45 Voir par exemple Il. XIII.726-735. 
la beauté, le courage et l'éloquence (v. 27-28). A l'opposé de cette gloire, Grégoire définit la sienne propre, qui repose «sur les souffrances» (v. 29).

L'effet de surenchère dans les souffrances apparaît aussi dans la sphragis du poème (v. 25-26). Grégoire semble ici imiter la signature de Nicandre qui écrivait :

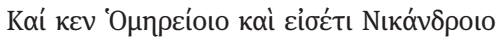

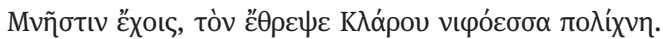

«Et, du poète homérique, tu pourras à jamais garder le souvenir, de Nicandre qu'éleva la blanche bourgade de Claros ${ }^{46}$.

S’il s'exprime comme le poète homérique, Grégoire ne définit toutefois pas sa ville d'origine de manière méliorative, mais en soulignant sa petitesse, avec l'adjectif óAíyn qui rappelle la dimension paradoxale de la gloire revendiquée.

En conclusion, l'analyse attentive de ces poèmes bibliques montre bien que ces pièces, en apparence courtes et banales, ont fait l'objet d'un travail d'écriture élaboré et présentent à ce titre un intérêt sur le plan littéraire. Elles constituent un bon exemple de la manière dont Grégoire entrelace deux traditions et réussit à créer une impression d'homogénéité et de cohérence. C'est sans doute parce que le poète favorise les motifs présents à la fois dans la Bible et la poésie épique et aussi parce qu'il procède par allusion discrète et savante. Par ailleurs, Grégoire essaie de marquer l'esprit du lecteur en mettant en scène de manière aussi frappante que possible les figures bibliques. Il reprend un lexique militaire adapté à son propos et des procédés épiques propres à renforcer l'expression, comme l'amplification, la surenchère, ou la stylisation. La figure centrale est celle de Jésus et c'est celle qui est la plus mise en valeur. Si peu de vers sont consacrés aux figures vétéro-testamentaires, ces personnages jouent toutefois un rôle important puisqu'ils servent de modèles héroïques à Grégoire et que c'est par leur intermédiaire que le poète se met en scène comme un héros épique au service de la Trinité. C'est sans aucun doute cet aspect qui fait l'originalité de la poésie de Grégoire : le poète met en effet en scène un «je» pas seulement épique mais aussi lyrique, devenant un anti-héros appelé à une gloire paradoxale et chantant ses propres malheurs.

\section{Bibliographie}

Demoen (1996): Kristofell Demoen, Pagan and biblical exempla in Gregory Nazianzen: a study in rhetoric and hermeneutics, Turnhout.

Cotter (1999): Wendy Cotter, Miracles in greco-roman Antiquity, Londres.

Nagy (1991): Gregory Nagy, The Best of the Acheans: Concepts of the Hero in Archaic Greek poetry, Baltimore.

46 Nicandre, Thériaques, v. 957-958, Paris, C.U.F., 2002 (trad. J.-M. Jacques, p. 75). 
Prudhomme (2006): Juliette Prudhomme, «L'oeuvre poétique de Grégoire de Nazianze: héritage et renouveau littéraires», 2006, thèse en ligne, p. 99-103 (http://www.theses.fr/ 2006LYO20054).

Vernant (1989): Jean Pierre Vernant, L'individu, la mort, l'amour, Paris. 
